\title{
Karyotype and reproduction mode of the rodent parasite Strongyloides venezuelensis
}

\author{
AKINA HINO ${ }^{1}$, TERUHISA TANAKA ${ }^{2}$, MAHO TAKAISHI ${ }^{1}$, YUMIKO FUJII ${ }^{1}$, \\ JUAN E. PALOMARES-RIUS ${ }^{1}$, KOICHI HASEGAWA ${ }^{3}$, HARUHIKO MARUYAMA ${ }^{1}$ \\ and TAISEI KIKUCHI ${ }^{1} *$ \\ ${ }^{1}$ Division of Parasitology, Faculty of Medicine, University of Miyazaki, Miyazaki, 889-1692 Japan \\ ${ }^{2}$ Department of Infections, Respiratory and Digestive Medicine, Faculty of Medicine, University of the Ryukyus, Okinawa, \\ 903-0213 Fapan \\ ${ }^{3}$ Department of Environmental Biology, College of Bioscience and Biotechnology, Chubu University, Kasugai, Aichi, \\ 487-8501 Fapan
}

(Received 14 April 2014; revised 9 May and 4 Fune 2014; accepted 8 Fune 2014; first published online 4 August 2014)

SUMMARY

Strongyloides venezuelensis is a parasitic nematode that infects rodents. Although Strongyloides species described to date are known to exhibit parthenogenetic reproduction in the parasitic stage of their life cycle and sexual reproduction in the freeliving stage, we did not observe any free-living males in $S$. venezuelensis in our strain, suggesting that the nematode is likely to depend on parthenogenetic reproduction. We confirmed by cytological analysis that $S$. venezuelensis produces eggs by parthenogenesis during the parasitic stage of its life cycle. Phylogenetic analysis using nearly the full length of 18S and D3 region of $28 \mathrm{~S}$ ribosomal RNA gene suggested that $S$. venezuelensis is distantly related to another rodent parasite, namely Strongyloides ratti, but more closely related to a ruminant parasite, Strongyloides papillosus. Karyotype analysis revealed $S$. venezuelensis reproduces with mitotic parthenogenesis, and has the same number of chromosomes as $S$. papillosus $(2 n=4)$, but differs from $S$. ratti $(2 n=6)$ in this regard. These results, taken together, suggest that $S$. venezuelensis evolved its parasitism for rodents independently from $S$. ratti and, therefore, is likely to have a different reproductive strategy.

Key words: parthenogenesis, parasitic nematodes, karyotype.

\section{INTRODUCTION}

The genus Strongyloides comprises over 50 species of nematodes that parasitize mammals, amphibians, reptiles and birds (Viney and Lok, 2007). Strongyloides nematodes have complex but interesting life cycles. Infection by Strongyloides begins when the infective third stage larvae (iL3) attach to and penetrate the host skin. Once inside the host, they moult twice into parasitic adults and settle in the small intestine of the host. Then, the parasitic adults, all female, produce eggs by parthenogenesis (Fig. 1A). Once the eggs or hatched larvae are excreted from the host, they develop via the homogonic route into iL3 forms or the heterogonic route into free-living stages that reproduce sexually outside the host (Fig. 1A); sexual reproduction in the free-living generation was shown for two species, Strongyloides ratti (Viney, 1996) and Strongyloides papillosus (Eberhardt et al. 2007). The progeny of free-living adults are entirely female and develop into iL3 forms in most Strongyloides species, including S. ratti and Strongyloides stercoralis, with a few exceptions (Yamada et al. 1991; Streit, 2008).

Parthenogenetic reproduction by parasitic females was found to be mitotic in cytological studies for

* Corresponding author: Division of Parasitology, Faculty of Medicine, University of Miyazaki, Miyazaki, 889-1692 Japan. E-mail: taisei_kikuchi@med.miyazaki-u.ac.jp
S. papillosus, Stronglyoides ransomi (Triantaphyllou and Moncol, 1977) and for S. ratti (Chitwood and Graham, 1940), and using molecular markers for S. ratti (Viney, 1994) and S. papillosus (Nemetschke et al. 2010). In these species, the progeny from parasitic females can develop into one of three distinct morphologies: free-living females, free-living males (both via the heterogonic route) or iL3s (homogonic route). Although the ratio of these morphologies is influenced by environmental factors such as host immune response, temperature and $\mathrm{pH}$ (Arizono, 1976; Moncol and Triantaphyllou, 1978; Nwaorgu, 1983; Viney, 1996; Harvey et al. 2000; Minato et al. 2008; Sakamoto and Uga, 2013), the detailed mechanisms which determine their route of development remain unclear. Moreover, S. stercoralis and S. ratti harbour three pairs of chromosomes, and one of them is a sex (X) chromosome. Females of these species have an XX and free-living males have an XO karyotype (Streit, 2008). Genetic material homologous to two chromosomes in $S$. ratti, namely I and X, appears combined into one chromosome in S. papillosus. Additionally, an XX/XO karyotype appears to be functionally restored in males by a chromatin diminution event (Nemetschke et al. 2010).

Strongyloides venezuelensis parasitizes rodent species including rats, mice and Mongolian gerbil Meriones unguiculatus and is distributed worldwide 
A

\section{Strongyloides ratti}

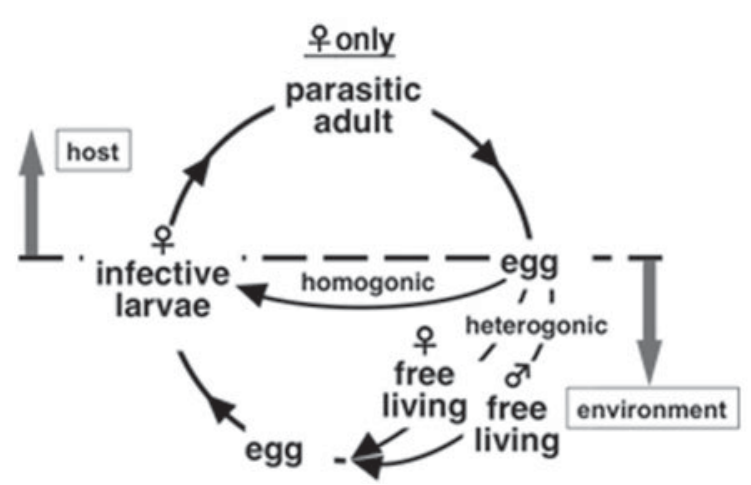

B

\section{Strongyloides venezuelensis}

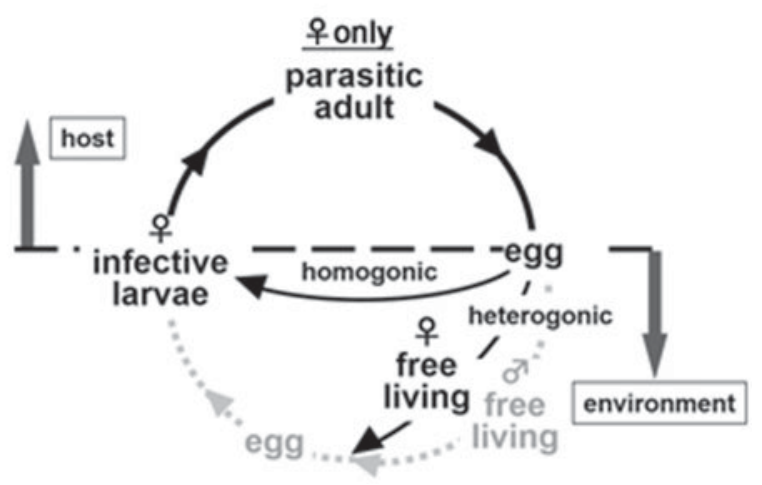

Fig. 1. (A) Life cycle of Strongyloides ratti. Parasitic females produce eggs by parthenogenesis in the host's small intestine and the eggs are excreted into the environment in the faeces. Eggs develop into infective larvae by two alternative routes. In the homogonic route, eggs develop directly into infective larva. In the heterogonic route, eggs develop into free-living forms, reproducing sexually, after which the resultant eggs develop into infective larva. (B) Life cycle of Strongyloides venezuelensis. S. venezuelensis may lack the heterogonic developmental route in its life cycle.

(Brumpt, 1934; Wertheim and Lengy, 1964; Little, 1966; Hasegawa et al. 1988). Strongyloides venezuelensis, alongside $S$. ratti, is one of the most widely used laboratory models to study Strongyloides infection and mucosal immunity (Sato and Toma, 1990; El-Malky et al. 2013). Although both S. ratti and $S$. venezuelensis have rodents as hosts, the individual mechanisms by which they establish parasitism are thought to differ from each other. Phylogenetic analysis with ribosomal RNA gene suggested that $S$. venezuelensis is not as closely related to $S$. ratti as to S. papillosus and the primate parasite Strongyloides fuelleborni (Dorris et al. 2002). The migration patterns of the two rodent parasites in the host (Takamure, 1995), as well as mechanisms of host immunomodulation for successful parasitism, differ from each other as well (Wilkes et al. 2007; Matsumoto et al. 2013). More interestingly, observation of free-living adults is less common in $S$. venezuelensis as compared with $S$. ratti (Hasegawa et al. 1988; Harvey et al. 2000), suggesting that reproduction strategies used by the two species may also be different. Thus, the parasitic abilities of the two species may have evolved independently from each other, and a comparison between the two may provide invaluable insights into understanding the parasitic mechanism and its evolution in Strongyloides nematodes.

Though S. venezuelensis is a widely used laboratory model, some aspects of its basic biology remain unclear. In this report, we re-examined the phylogenetic position of $S$. venezuelensis within the genus Strongyloides, using 18S rRNA and the D3 expansion segment of $28 \mathrm{~S}$ rRNA genes. We also examined the developmental routes of progeny from the parasitic females, chromosome behaviour in the germ cells and early embryos in S. venezuelensis. Our analyses suggest that acquisition of rodent parasitism occurred independently in S. venezuelensis and S. ratti.

\section{MATERIALS AND METHODS}

\section{Culturing and handling nematodes}

Strongyloides venezuelensis $\mathrm{HH} 1$ and $S$. ratti TDI isolates were used in this study. They were isolated from Okinawa, Japan (Hasegawa et al. 1988) and Tokyo, Japan, respectively, and maintained in the Parasitology laboratory of the University of Miyazaki, using male Wistar rats. Infectious aliquots were prepared by faecal culture using filter paper at $27^{\circ} \mathrm{C}$ for 2 days and 5 days for S. venezuelensis and $S$. ratti, respectively (Sato and Toma, 1990). The nematodes were washed three times in distilled water, and administered by subcutaneous injection. Strongyloides papillosus iL3 was supplied from Dr Ayako Yoshida of University of Miyazaki. Parastrongyloides trichosuri DNA (strain KNP from Warwick Grant's lab) was supplied by Berriman lab from Wellcome Trust Sanger Institute, UK. Strongyloides stercoralis was collected in Yangon, Myanmar.

\section{PCR conditions and DNA sequencing}

Nearly full-length $18 \mathrm{~S}$ ribosomal DNA (rDNA) was amplified with primers $988 \mathrm{~F}-1912 \mathrm{R}$ and $1813 \mathrm{~F}-$ 2646R from a lysate of a single nematode as described previously (Holterman et al. 2006). These primers amplify $1652 \mathrm{bp}$ out of $1754 \mathrm{bp}$ of full-length $18 \mathrm{~S}$ rDNA of C.elegans (NR_000053·1). The D3 region of $28 \mathrm{~S}$ rDNA was amplified using primers D3A-D3B 
(Nunn et al. 1996). PCR amplifications were carried out in $30 \mu \mathrm{L}$ reaction mixtures containing $15 \mu \mathrm{L}$ GoTaq Green Master Mix (Promega), 0.5 $\mathrm{M}$ of each primer, and $1 \mu \mathrm{L}$ of appropriately diluted nematode lysate under thermal-cycling conditions of $94^{\circ} \mathrm{C}$ for $1 \mathrm{~min}$, followed by 30 cycles of $94^{\circ} \mathrm{C}$ for $30 \mathrm{~s}, 53^{\circ} \mathrm{C}$ for $30 \mathrm{~s}$ and $72^{\circ} \mathrm{C}$ for $1 \mathrm{~min}$. PCR products were purified before sequencing using a MinElute $96 \mathrm{UF}$ PCR purification plate (QIAGEN). DNA sequencing was performed using the BigDye Terminator 3.1 kit and ABI PRISM 3700 or 3130 Genetic Analyzer (Applied Biosystems).

\section{Phylogenetic analyses}

Nearly full-length 18S rRNA gene and D3 expansion segments of 28S rRNA gene of Strongyloides species were used for phylogenetic reconstruction. Parastrongyloides trichosuri was used as an outgroup taxon. The newly obtained and published sequences for each gene were aligned using MAFFT (Katoh et al. 2002) with iterative refinement method (FFT-NS-i). Both datasets were concatenated using Concatenator v.1.1.0. (Pina-Martins and Paulo, 2008). Phylogenetic analyses of the sequence dataset were performed with maximum likelihood (ML) using PAUP*4b10 (Wilgenbusch and Swofford, 2003) and Bayesian inference (BI) using MrBayes 3.1.2 (Huelsenbeck and Ronquist, 2001). The bestfitted model of DNA evolution was obtained using ModelTest v.2 (Darriba et al. 2012) with the Akaike Information Criterion (AIC). The Akaike-supported model, base frequency, proportion of invariable sites, gamma distribution shape parameters and substitution rates in the AIC were then used in phylogenetic analyses. BI analysis under the TVM+G model for D3 expansion segment of 28S and the $\mathrm{TVM}+\mathrm{I}+\mathrm{G}$ model for $18 \mathrm{~S}$ rDNA was initiated with a random starting tree and run with four Metropoliscoupled Markov chain Monte Carlo (MCMC) for $1 \times 10^{6}$ generations in the combined dataset. The MCMC were sampled every 100 generations. Two runs were performed for each analysis. After discarding burn-in samples and evaluating convergence, the remaining samples were retained for further analyses. The topologies were used to generate a $50 \%$ majority rule consensus tree. Trees were visualized using TreeView (Page, 1996). In ML analysis the estimation of the support for each node was obtained by bootstrap analysis with 1000 heuristic search replicates using the previously obtained models. Posterior probabilities and bootstrap support are given on respective clades.

\section{Free-living occurrence observation}

Three rats were infected with 500 iL 3s of $S$. venezuelensis or S. ratti. Faeces samples from each rat were collected at 8, 12, 14, 16 and 24 days post-infection (d.p.i.) for $S$. venezuelensis and at $8 \mathrm{~d}$. p.i. for S. ratti. Faeces (approx. $0.5 \mathrm{~g}$ ) were cultured on a $2 \%(\mathrm{w} / \mathrm{v})$ agar plate at various temperatures $\left(19,25\right.$, or $\left.30^{\circ} \mathrm{C}\right)$ for 3 days and free-living male and female nematodes were counted. Three agar plates for each day and each condition were used. A portion of the faecal samples $(c .1 .5 \mathrm{~g})$ was diluted in distilled water to determine the eggs/larvae per gram (epg/lpg) of faeces. All statistical analyses were performed using the software package $\mathrm{R}$ version 2.15 .2 (http:// www.r-project.org).

\section{Early embryogenesis}

Parasitic females were obtained from rat intestine at 5-10 d.p.i. Fresh parasites were transferred to a pre-warmed $\left(37^{\circ} \mathrm{C}\right)$ agarose pad $(4 \%(\mathrm{w} / \mathrm{v})$ agarose) prepared on a microscope slide (Shaham, 2006) and covered with a silicon grease-rimmed cover slip and viewed under a Nomarski microscope (IX71, Olympus, Japan). All procedures involving the microscopic observations were performed at $37^{\circ} \mathrm{C}$.

\section{DAPI staining/microscopy}

Parasitic females were fixed with ice-cold methanol and stained with DAPI as previously described (Nemetschke et al. 2010). To collect the eggs, parasitic females were allowed to lay eggs in PBS, the eggs were then squashed and stained as previously described with the exception of using methanol instead of acetic acid for fixation (Albertson et al. 1979). Microscopic observations were carried out using a confocal laser scanning microscope (LSM700, Zeiss).

\section{RESULTS}

\section{Phylogenetic position of $\mathrm{S}$. venezuelensis}

In order to determine the phylogenetic position of $S$. venezuelensis in the genus, we used nearly fulllength 18S rRNA and D3 expansion segments of $28 \mathrm{~S}$ rRNA genes. We sequenced these regions in $S$. venezuelensis, S. papillosus, S. stercoralis, S. ratti and $P$. trichosuri for phylogenetic analysis. $18 \mathrm{~S}$ sequences from other Strongyloides species obtained from the public database were also included in the analysis. Our phylogenetic tree using P. trichosuri as an outgroup indicated that the Strongyloides species can be divided into two clades (Fig. 2): one clade comprising of S. papillosus, S. fuelleborni, $S$. venezuelensis, Strongyloides callosciureus, Strongyloides robustus and Strongyloides cebus, and the other including S. stercoralis, Strongyloides procyonis and S. ratti. Within the former clade, $S$. venezuelensis was further clustered into a subclade with S. papillosus and S. fuelleborni. 


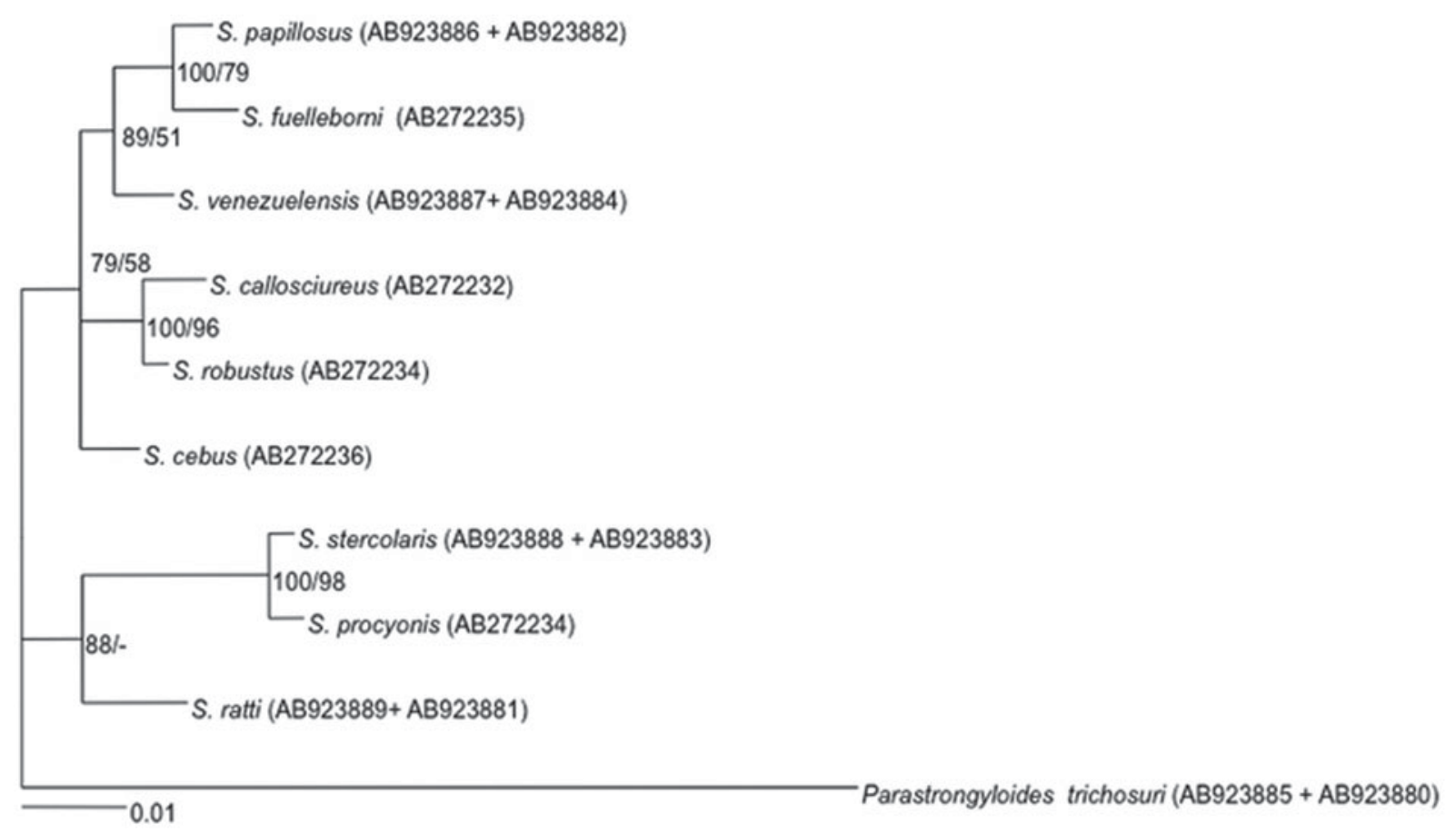

Fig. 2. The molecular phylogenetic relationship between Strongyloides species inferred from concatenated nearly full-length 18S rRNA gene (1640 positions from edited alignment) and D3 expansion segments of 28S rRNA gene (289 positions from edited alignment). TVM $+\mathrm{G}$ and TVM $+\mathrm{I}+\mathrm{G}$ were used for D3 expansion segment of $28 \mathrm{~S}$ and $18 \mathrm{~S}$, respectively. Posterior probabilities (first number in the node label) more than $65 \%$ are given for appropriate clades; bootstrap values greater than $50 \%$ are given on appropriate clades in ML analysis (second number in the node label).

Occurrences of free-living adults in $\mathrm{S}$. venezuelensis

As the ratio of free-living females/males $v s$ females developing homogonically into iL $3 \mathrm{~s}$ is influenced by several factors, including host immunity and other environmental conditions (temperature, $\mathrm{pH}$ etc.), in other Strongyloides species (Arizono, 1976; Moncol and Triantaphyllou, 1978; Nwaorgu, 1983; Viney, 1996; Harvey et al. 2000; Minato et al. 2008; Sakamoto and Uga, 2013), we used two parameters (days post infection and faeces incubation temperature) to examine the occurrence of free-living nematodes (FLNs) in S. venezuelensis.

In a total of 162 attempts, comprising of six d.p.i.s $(8,12,14,16,20$ and 24) and three temperature conditions $\left(19,25\right.$ and $\left.30^{\circ} \mathrm{C}\right)$, each with three rats and triplicate, we observed FLNs of $S$. venezuelensis in only 19 cases. As this frequency is much lower than that reported in S. ratti (Harvey et al. 2000; Minato et al. 2008; Sakamoto and Uga, 2013), we validated our method using $S$. ratti in place of $S$. venezuelensis. In the $S$. ratti experiment we observed FLNs in all culture plates tested (Table S1) and the numbers of FLNs (8.32 to $17 \cdot 4$ FLNs per 1000 eggs) were similar to those reported previously (Minato et al. 2008). Therefore we confirmed that this low frequency (19 out of 162 cases) was not due to errors in handling of the samples. Of note is that in some culture plates (52 cases) we did not observe the homogonically developed nematodes (iL3s) although the faeces contained a sufficient number of eggs, which may suggest that the development of $S$. venezuelensis is more sensitive to environmental conditions than that of $S$. ratti. Furthermore, free-living males were never detected in $S$. venezuelensis (Table 1) while approximately half of FLNs in the $S$. ratti experiment were males (Table S1). Consequently, no hatching was observed in eggs derived from the free-living females (data not shown).

In the 19 plates with $S$. venezuelensis FLNs, 7 plates $\left(17 \mathrm{FLNs}\right.$ in total) were from $25^{\circ} \mathrm{C}$ culture and 12 plates (46 FLNs) were from $30^{\circ} \mathrm{C}$ culture (Table 1). We did not observe any FLNs in $19^{\circ} \mathrm{C}$ cultures ('Table 1).

We observed S. venezuelensis FLNs in 1, 3, 4, 3, 2, and 2 cases at 8, 12, 14, 16, 20 and 24 d.p.i., respectively (Fig. 3 ) and the number of FLNs on each d.p.i. was $3,6,6,43,4$, and 4, respectively. Although the number of eggs per gram of faeces (epg) decreased as the d.p.i. increased (Fig. 3), the number of instances that FLNs were observed did not change significantly (Generalized Linear Model with binomial error distribution: $\mathrm{d} f=52, P>0.9)$. Then, we sought to investigate the relationship between epg and occurrence of FLNs. Figure S1 shows a plot of epg and number of FLNs per gram of faeces. The trend observed was that fewer epg showed more numbers of FLNs per egg (Fig. S1).

The highest number of FLNs was obtained with faeces collected at 16 d.p.i. and cultured at $30^{\circ} \mathrm{C}$ 
Table 1. Total number of free living nematodes observed in S. venezuelensis faeces samples

\begin{tabular}{llcc}
\hline \hline & $19{ }^{\circ} \mathrm{C}$ & $25{ }^{\circ} \mathrm{C}$ & $30{ }^{\circ} \mathrm{C}$ \\
\hline Free-living female & $0(0 / 54)$ & $17(7 / 54)$ & $46(12 / 54)$ \\
Free-living male & $0(0 / 54)$ & $0(0 / 54)$ & $0(0 / 54)$ \\
\hline \hline
\end{tabular}

Fifty-four culture plates were used at each temperature. Numbers in parentheses represent numbers of incidents of free-living observation (i.e. 17(7/54) indicates a total number of 17 free-living nematodes found in 7 out of 54 culture plates).

(34 FLNs, 6.58 per 1000 eggs). However this was an outlier as the other two plates under the same conditions (same rat, d.p.i. and temperature) showed much lower numbers of FLNs $(0 \cdot 24$ or 0 per 1000 eggs).

\section{Germ cells in the parasitic female}

Strongyloides venezuelensis parasitic females have two elongated (didelphic) gonads extending from the vulva to the head or the tail directions, reflexed around the beginning of the intestine or anus, continuing around the vulva (Little, 1966) (Fig. 4A, B). Egg development and embryogenesis progress as stages move along the tract from the germinal tissue to the vulva. Both gonads spiral about themselves around the intestine (Fig. 4A, B, E). As with many nematodes the distal part of the germ line was a syncytium; nuclei were located at the inner surface of the germ line (Fig. 4C-E). Chromosomes in all nuclei in the distal region dispersed peripherally, and nuclei were condensed in the 'germinal zone' (Fig. 4F). As nuclei moved away from the germinal zone they were packed one by one into a cell in the 'growth zone' and became oocytes (Fig. 4G). Germinal vesicles appeared and chromosomes became condensed during the oocyte movement towards the proximal region, and they seemed to be arrested at this stage until they passed through the oviduct (Fig. 4G). We detected four chromosomes and this state could be prometaphase of the 'maturation division' (see below). We did not identify any sperm or sperm nuclei in the gonad in either bright-field or DAPI-stained observations. From these results, we conclude that parasitic females of $S$. venezuelensis reproduce parthenogenetically.

\section{Early embryogenesis of eggs from the parasitic female}

To see if the embryo starts development without fertilization, we observed oocyte maturation and early embryogenesis in the parasitic female sequentially with light microscopy. After the mature oocyte passed through the oviduct into the uterus, the eggshell was formed (Fig. 5A). The embryo shape was oval and the long axis of the embryo in the uterus

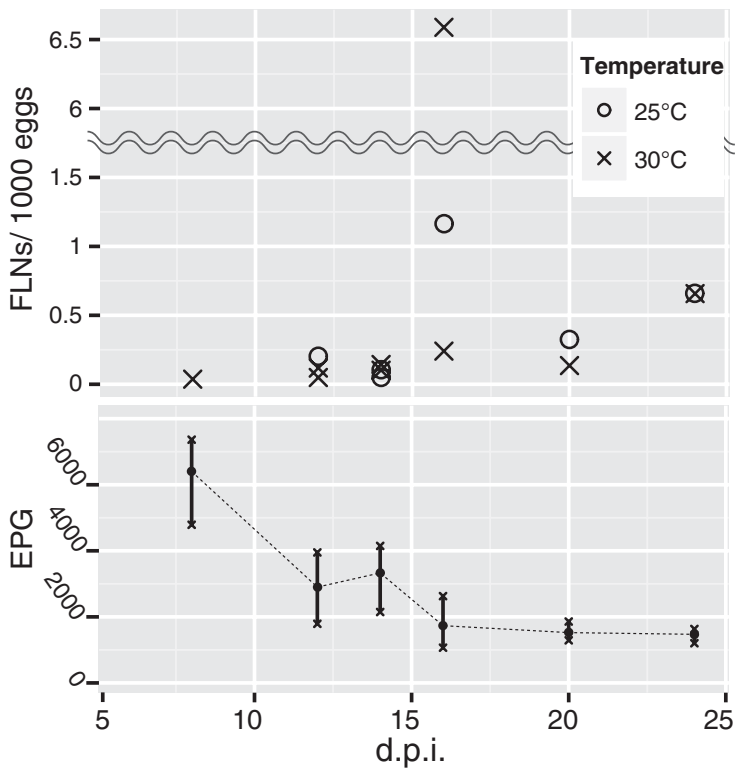

Fig. 3. (Upper) Free-living occurrences in $S$. venezuelensis. Nineteen positive cultures (in which free-living nematodes were found) out of 162 were plotted by number of free-living nematodes (FLNs) per 1000 eggs and day post infection (d.p.i.). Faeces were cultured at 19,25 or $30^{\circ} \mathrm{C}$. No free-living nematodes were observed in $19^{\circ} \mathrm{C}$ cultures. (Bottom) EPG change by d.p.i. The black dot represents the mean and crosses represent the highest and the lowest in each d.p.i.: $n=3$.

was in parallel to the anterior-posterior axis of the mother. The germinal vesicle disappeared, and then only one pronucleus appeared at the lagging side-pole of the embryo (Fig. 5A, B). At this stage a protruded polar body-like structure was observed adjacent to the pronucleus (Fig. 5B). Pseudocleavage furrow and cytoplasmic streaming were observed (Fig. 5C), the pronucleus moved towards the middle of the cell (Fig. 5D) and its membrane broke down (Fig. 5E, F). Subsequently the embryo divided to form the two-cell stage (Fig. 5G, H) and the four-cell stage (Fig. 5I). All embryos $(n=7)$ observed in these experiments developed to first-stage larvae and hatched successfully (not shown), suggesting that the process observed was normal development.

\section{Chromosome structure and behaviour during early embryogenesis}

To clarify the mechanism by which ploidy is maintained, we observed chromosome structure and behaviour during early embryogenesis by DAPI staining. Developmental stages of the fixed and stained embryos were decided by comparison to the living embryos observed above. After passing through the oviduct into the uterus, the oocyte resumed mitotic cell division (Fig. 6A). This division always occurred asymmetrically at the lagging side-pole of the embryo (Fig. 6A) to produce one polar body. Polar bodies stained clearly as blue dots, but often disappeared 


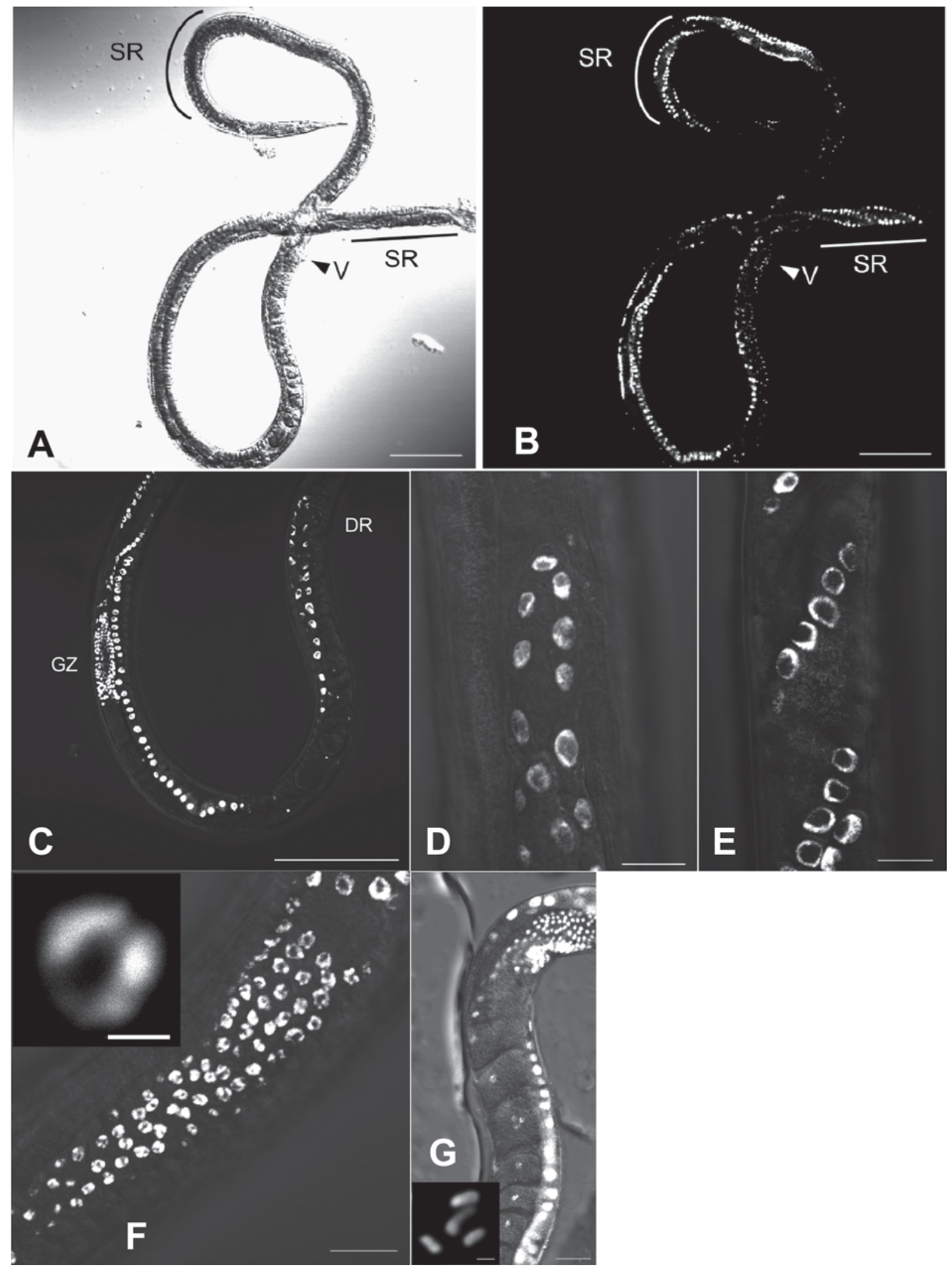

Fig. 4. Germ cells in a parasitic female of $S$. venezuelensis. (A, B) Whole body of a parasitic female under bright field and DAPI fluorescence microscopy. V, vulva; SR, spiralled region; (C) Distal side of a gonad. DR: distal region GZ: germinal zone; (D) Oogonium in distal end of a gonad; (E) Oogonium in spiralled region of a gonad; (F) Condensed nucleus in the germinal zone; $(\mathrm{G})$ Condensed chromosomes in the growth zone. (Scale bars: A, B, C = 100 $\mu \mathrm{m}$; D, E, $\mathrm{F}=10 \mu \mathrm{m} ; \mathrm{G}=20 \mu \mathrm{m}$; boxes in $\mathrm{F}, \mathrm{G}=1 \mu \mathrm{m})$. 

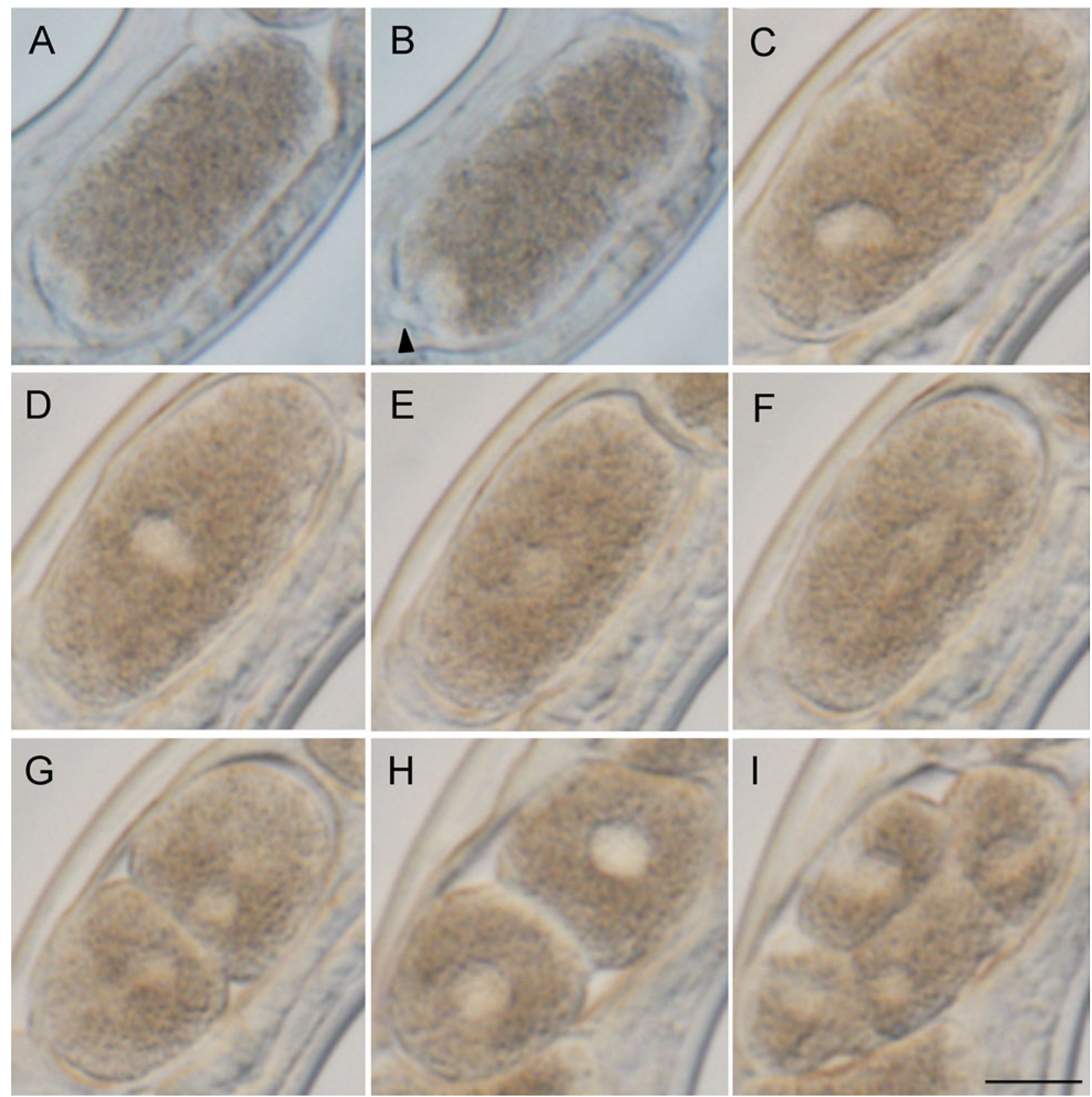

Fig. 5. Early embryogenesis in a live S. venezuelensis parasitic female. The leading edge of the embryo is arranged to be upper right. (A) An embryo that has passed through the oviduct; (B) An embryo under maturation division; (C) Pseudocleavage with one pronucleus; (D) Nucleus moving to the centre of the cell; (E) Nucleus dispersed at the centre of the cell; (F, G, H) Cell division to form a two-cell embryo; (I) Four-cell embryo (Arrow head indicates a polar body, Scale bar $=10 \mu \mathrm{m})$. Time frame of the development is shown in Table S2.

as embryogenesis progressed. One pronucleus was reconstructed with vesicle (Fig. 6B), moved to the centre, and chromosomes were visible in the prophase stage (Fig. 6C). Chromatids fused and aligned along the metaphase plate (Fig. 6D) and migrated to each pole (Fig. 6E) subsequently forming two-cell stage embryos (Fig. 6F). The cleavage occurred asymmetrically with a bigger blastomere in the leading pole than the lagging one, which is contrary to the observations in Caenorhabditis elegans (Wallenfang and Seydoux, 2000) and other Caenorhabditis nematodes (Brauchle et al. 2009). Most eggs were laid as two-cell stage embryos. Then, we collected the laid eggs, squashed and stained with DAPI. Four chromosomes, two longer than the others, were observed in prophase cells in the four-cell stage embryos (Fig. 6G, H), suggesting that the karyotype of $S$. venezuelensis is $2 n=4$. We confirmed in nine eggs, all of which had four chromosomes, and no specimens showed different compositions of chromosomes.

\section{DISCUSSION}

Strongyloides spp. are unique among parasitic nematodes in having both parasitic and free-living stages 

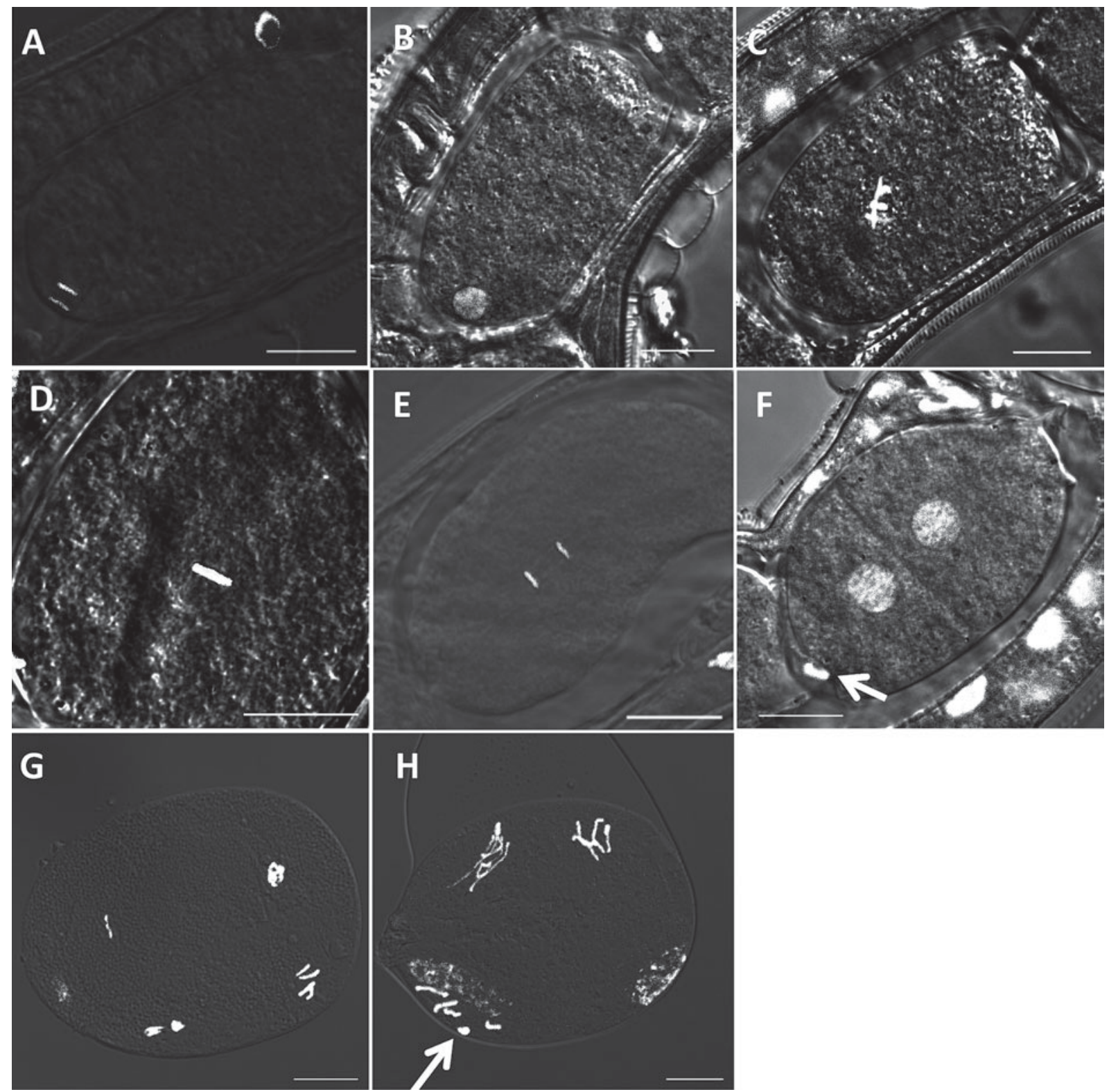

Fig. 6. Chromosome behaviour after passing through oviduct into the uterus. The leading edge of the embryo is arranged to be upper right. (A) Anaphase of maturation division; (B) A pronucleus reconstructed after maturation division; (C) A pronucleus moves to the centre and chromosomes are formed; (D) Metaphase of the first embryonic cell division; (E) Anaphase of the first embryonic cell division; (F) Telophase of the first embryonic cell division; $(\mathrm{G}, \mathrm{H})$ Four cell embryos. Newly laid eggs were squashed and stained for the observation. (Arrows indicate polar bodies. All scale bars $=10 \mu \mathrm{m}$ ).

in their life cycle. Since the parasitic females produce eggs by parthenogenesis, the free-living stage is the only occasion for them to exchange genetic materials with other individuals. Eggs that are parthenogenetically produced by the parasitic females can develop into three morphs: free-living males, free-living females or iL3. In S. ratti the frequency of free-living development is as high as $60 \%$ (Minato et al. 2008), which suggests frequent exchanges of genetic materials take place in this species. In this study we observed a much lower frequency of free-living development in S. venezuelensis than $S$. ratti. Furthermore, we did not find any freeliving males. This suggests that $S$. venezuelensis rarely, or possibly never, exchanges genetic materials with other individuals in its life cycle. It is still possible, however, that there are unknown triggers to stimulate the nematode to have more free-living males and enable them to perform genetic exchanges. This could be an artificial loss of ability due to the stress of prolonged maintenance in laboratory, though absence of free-living males was reported previously in a recent field isolate of $S$. venezuelensis (Hasegawa et al. 1988). It would be of value to investigate free-living occurrence rates in other strains or wild isolates to confirm if this is a general characteristic of the species or the observation in this study is an exceptional case. 
We noted that the number of free-living females increased with days after infection, although it was not clear which parameter was more influential, days post infection (i.e. ageing of worms and effect of host immunity) or dispersed density of eggs in the faeces. We also observed a higher number of free-living females in 30 and $25^{\circ} \mathrm{C}$ cultures than at $19^{\circ} \mathrm{C}$. A similar trend for temperature conditions was also observed with other Strongyloides species (Arizono, 1976; Nwaorgu, 1983; Minato et al. 2008; Sakamoto and Uga, 2013).

We observed four chromosomes in S. venezuelensis somatic cells, suggesting $S$. venezuelensis has a karyotype of $2 n=4$. It is known that $S$. ratti has three pairs of chromosomes $(2 n=6)$ and one of them is a sex chromosome. On the other hand, S. papillosus females have two pairs of chromosomes $(2 n=4)$. The lengths of the two are significantly different and the longer one is suggested to be the result of a fusion of a sex chromosome with one of the autosomes of $2 n=6$ (Triantaphyllou and Moncol, 1977). The number of chromosomes in $S$. venezuelensis could also result from such a fusion. However, the lengths of the two pairs of the four S. venezuelensis chromosomes did not differ from each other to the extent seen in S. papillosus (Nemetschke et al. 2010). It would be very interesting to correlate the evolutionary history of reproduction in Strongyloides species with their chromosome structures.

Nemetschke et al. showed that S. papillosus employs chromatin diminution to exclude chromosome regions corresponding to the $S$. ratti sex chromosome in males (Albertson et al. 1979; Nemetschke et al. 2010). Although S. venezuelensis has the same number of chromosomes as $S$. papillosus and chromosome diminution could generate males in this species, we did not observe such events or any different chromosome composition in this study. This correlates with the absence of males in S. venezuelensis, although the basis for the lack of chromatin diminution is unclear: it could be that we were unable to generate $S$. venezuelensis males via this procedure or that males were not observed because $S$. venezuelensis is not capable of chromatin diminution.

Because embryogenesis occurred without fertilization and the chromosome number in mature oocytes, which are located proximal to the oviduct, was the same as that of the somatic cells it is likely the $S$. venezuelensis reproduces via mitotic parthenogenesis. Even without sperm stimulation, an embryo of S. venezuelensis had a polarity and produced the polar body on the lagging pole of the embryo. This orientation is opposite from $C$. elegans (Wallenfang and Seydoux, 2000). Parasitic stages of Strongyloides species including $S$. ratti or $S$. papillosus have been reported to have only female sex and reproduce by mitotic parthenogenesis (Zaffagnini, 1973; Triantaphyllou and Moncol, 1977; Viney, 1994). Our observation is consistent with these reports.
In order to confirm this, molecular studies would be useful by checking the progenies have the same genotype (clones) or have genetic variations due to cross-overs of sister chromosomes.

Our phylogenetic analysis using genes for $18 \mathrm{~S}$ and D3 of 28S ribosomal RNA showed two wellsupported clusters in Strongyloides species, namely a group including $S$. ratti and $S$. stercoralis and another including $S$. papillosus and $S$. venezuelensis (Fig. 2). This is consistent with the gonad morphologies of these species, wherein members of the $S$. papillosus group have spiral morphology, while those of the $S$. ratti group have straight morphology (Bartlett, 1995; Little, 1966; Sato et al. 2007). The number of chromosomes observed for Strongyloides species was also consistent with the phylogeny; karyotypes known thus far are $2 \mathrm{n}=6$ for $S$. ratti and $S$. stercoralis and $2 \mathrm{n}=4$ for $S$. papillosus and $S$. venezuelensis. Strongyloides ratti and $S$. venezuelensis are both parasites of rodents. However they are clearly different in terms of rRNA phylogeny, chromosome number and gonad morphology. Therefore, it is likely that they acquired their ability to parasitize rodents independently from each other.

\section{SUPPLEMENTARY MATERIAL}

To view supplementary material for this article, please visit http://dx.doi.org/S0031182014001036.

\section{ACKNOWLEDGEMENTS}

The authors thank Mark Viney and Yasunobu Maeda for reading and commenting on the manuscript, Nancy Holroyd, Matthew Berriman, Warwick Grant, Ayako Yoshida and Yoichiro Horii for supplying DNA or other biological materials, and Valerie Williamson for valuable suggestions about embryo observation. This study was partly supported by JSPS KAKENHI [grant numbers 24780044, 24659190, 26292178 and 26460510]. AH was supported by the Association for Preventive Medicine of Japan.

\section{DATA DEPOSITION}

Sequence data has been deposited in GenBank/EMBL/ DDBJ under accession numbers AB923880-923889.

\section{REFERENCES}

Albertson, D. G., Nwaorgu, O. C. and Sulston, J. E. (1979). Chromatin diminution and a chromosomal mechanism of sexual differentiation in Strongyloides papillosus. Chromosoma 75, 75-87.

Arizono, N. (1976). Studies on the free-living generations of Strongyloides planiceps, 1943 II. Effect of temperature on the developmental types. Fapanese Fournal of Parasitology 25, 328-335.

Bartlett, C. M. (1995). Morphology, homogonic development, and lack of a free-living generation in Strongyloides robustus (Nematoda, Rhabditoidea), a parasite of North American sciurids. Folia Parasitologica 42, 102-114. Brauchle, M., Kiontke, K., Macmenamin, P., Fitch, D.H. and Piano, F. (2009). Evolution of early embryogenesis in rhabditid nematodes. Developmental Biology 335, 253-262.

Brumpt, E. (1934). Précis de Parasitologie, 6th Edn. Masson et Cie, Paris. 
Chitwood, B. and Graham, G. (1940). Absence of vitelline membranes on developing eggs in parasitic females of Strongyloides ratti. Fournal of Parasitology 26, 183-190.

Darriba, D., Taboada, G. L., Doallo, R. and Posada, D. (2012). jModelTest 2: more models, new heuristics and parallel computing. Nature Methods 9, 772.

Dorris, M., Viney, M. E. and Blaxter, M. L. (2002). Molecular phylogenetic analysis of the genus Strongyloides and related nematodes. International fournal for Parasitology 32, 1507-1517.

Eberhardt, A. G., Mayer, W.E. and Streit, A. (2007). The free-living generation of the nematode Strongyloides papillosus undergoes sexual reproduction. International Fournal for Parasitology 37, 989-1000.

El-Malky, M. A., Maruyama, H., Al-Harthi, S. A., El-Beshbishi, S. N. and Ohta, N. (2013). The role of B-cells in immunity against adult Strongyloides venezuelensis. Parasites and Vectors 6, 148.

Harvey, S. C., Gemmill, A. W., Read, A.F. and Viney, M. E. (2000). The control of morph development in the parasitic nematode Strongyloides ratti. Proceedings of the Royal Society B: Biological Sciences 267, 2057-2063.

Hasegawa, H., Orido, Y., Sato, Y. and Otsuru, M. (1988). Strongyloides venezuelensis Brumpt, 1934 (Nematoda: Strongyloididae) collected from Rattus norvegicus in Naha, Okinawa, Japan. Fapanese Fournal of Parasitology 37, 429-434.

Holterman, M., Van Der Wurff, A., Van Den Elsen, S. Van Megen, H., Bongers, T., Holovachov, O., Bakker, J. and Helder, J. (2006). Phylum-wide analysis of SSU rDNA reveals deep phylogenetic relationships among nematodes and accelerated evolution toward crown clades. Molecular Biology and Evolution 23, 1792-1800.

Huelsenbeck, J.P. and Ronquist, F. (2001). MrBayes: Bayesian inference of phylogenetic trees. Bioinformatics 17, 754-755.

Katoh, K., Misawa, K., Kuma, K. and Miyata, T. (2002). MAFFT: a novel method for rapid multiple sequence alignment based on fast Fourier transform. Nucleic Acids Research 30, 3059-3066.

Little, M. D. (1966). Comparative morphology of six species of Strongyloides (Nematoda) and redefinition of the genus. Fournal of Parasitology 52, 69-84.

Matsumoto, M., Sasaki, Y., Yasuda, K., Takai, T., Muramatsu, M., Yoshimoto, T. and Nakanishi, K. (2013). IgG and IgE collaboratively accelerate expulsion of Strongyloides venezuelensis in a primary infection. Infection and Immunity 81, 2518-2527.

Minato, K., Kimura, E., Shintoku, Y. and Uga, S. (2008). Effect of temperature on the development of free-living stages of Strongyloides ratti. Parasitology Research 102, 315-319.

Moncol, D. J. and Triantaphyllou, A. C. (1978). Stronglyoides ransomi: factors influencing the in vitro development of the free-living generation. Fournal of Parasitology 64, 220-225.

Nemetschke, L., Eberhardt, A. G., Hertzberg, H. and Streit, A. (2010). Genetics, chromatin diminution, and sex chromosome evolution in the parasitic nematode genus Strongyloides. Current Biology 20, 16871696.

Nunn, G. B., Theisen, B. F., Christensen, B. and Arctander, P. (1996) Simplicity-correlated size growth of the nuclear 28S ribosomal RNA D3 expansion segment in the crustacean order Isopoda. Fournal of Molecular Evolution 42, 211-223.
Nwaorgu, O. C. (1983). The development of the free-living stages of Strongyloides papillosus. I. Effect of temperature on the development of the heterogonic and homogonic nematodes in faecal culture. Veterinary Parasitology 13, 213-223.

Page, R. D. (1996). TreeView: an application to display phylogenetic trees on personal computers. Computer Applications in the Biosciences 12, 357-358.

Pina-Martins, F. and Paulo, O. S. (2008). Concatenator: sequence data matrices handling made easy. Molecular Ecology Resources 8, 1254-1255.

Sakamoto, M. and Uga, S. (2013). Development of free-living stages of Strongyloides ratti under different temperature conditions. Parasitology Research 112, 4009-4013.

Sato, H., Torii, H., Une, Y. and Ooi, H. K. (2007). A new rhabditoid nematode species in Asian sciurids, distinct from Strongyloides robustus in North American sciurids. Fournal of Parasitology 93, 1476-1486.

Sato, Y. and Toma, H. (1990). Effects of spleen cells and serum on transfer of immunity to Strongyloides venezuelensis infection in hypothymic (nude) mice. International fournal for Parasitology 20, 63-67.

Shaham, S. (2006). Methods in Cell Biology. WormBook, The C. elegans Research Community. doi: 10.1895/wormbook.1.49.1.

Streit, A. (2008). Reproduction in Strongyloides (Nematoda): a life between sex and parthenogenesis. Parasitology 135, 285-294.

Takamure, A. (1995). Migration route of Strongyloides venezuelensis in rodents. International Fournal for Parasitology 25, 907-911.

Triantaphyllou, A. C. and Moncol, D. J. (1977). Cytology, reproduction, and sex determination of Strongyloides ransomi and S. papillosus. Fournal of Parasitology 63, 961-973.

Viney, M. E. (1994). A genetic analysis of reproduction in Strongyloides ratti. Parasitology 109, 511-515.

Viney, M. E. (1996). Developmental switching in the parasitic nematode Strongyloides ratti. Proceedings of the Royal Society B: Biological Sciences, 263, 201-208.

Viney, M. E. and Lok, J. B. (2007). Strongyloides spp. WormBook 1-15. doi: 10.1895/wormbook.1.141.1.

Wallenfang, M. R. and Seydoux, G. (2000). Polarization of the anteriorposterior axis of C. elegans is a microtubule-directed process. Nature $\mathbf{4 0 8}$, 89-92.

Wertheim, G. and Lengy, J. (1964). The seasonal occurrence of Strongyloides ratti sandground, 1925 and of S. Venezuelensis Brumpt, 1934 in a population of Rattus norvegicus. Fournal of Helminthology 38, 393-398.

Wilgenbusch, J. C. and Swofford, D. (2003). Inferring evolutionary trees with PAUP*. Current Protocols in Bioinformatics, Chapter 6, Unit 6.4 doi: 10.1002/0471250953.bi0604s00.

Wilkes, C.P., Bleay, C., Paterson, S. and Viney, M. E. (2007). The immune response during a Strongyloides ratti infection of rats. Parasite Immunology 29, 339-346.

Yamada, M., Matsuda, S., Nakazawa, M. and Arizono, N. (1991). Species-specific differences in heterogonic development of serially transferred free-living generations of Strongyloides planiceps and Strongyloides stercoralis. Fournal of Parasitology 77, 592-594.

Zaffagnini, F. (1973). Parthenogenesis in the parasitic and free-living forms of Strongyloides papillosus (Nematoda, Rhabdiasoidea). Chromosoma 40, 443-450. 